\title{
Small Horizontal Angles and the Accuracy of Transit Lines
}

\author{
Leonard Eyges
}

I. InTRODUCTION. The measurement of the horizontal angle $A$ between two landmarks implies a circle of position (COP) somewhere along which the observer must lie. 'In an article in Navigation $\mathrm{News}^{2}$ a variety of qualitative aspects and practical uses of these circles has been discussed for the case when the angle is small, say less than $10^{\circ}$. A difficulty with small angle COPs is that the radius, when drawn on a chart, can become very large, of the order of metres, and the centre very distant, so that the circle cannot be drawn with a conventional drafting compass of reasonable size. In this note this difficulty is overcome by the derivation of a formula for plotting such circles as offsets from the transit line between the two objects. The formula is also applied to the question of the accuracy that can be achieved with transit lines of position.

2. THE SMALL-ANGLE COP AS OFFSETS FROM THE TRANSIT LINE. Figure I shows two landmarks, the transit line between them, and an arc of a small angle COP. As an aid to intuition we note that the arc shown has arbitrarily been taken to be that of a $5^{\circ}$ circle. We want to be able to draw such arcs for any angle without having to draw the circle as a whole. To this end let $H$ in the figure be the offset, the perpendicular distance from the transit line to the circle at a distance $D$ from the nearer landmark. The arc of the circle is defined by the following formula, giving $H$ as a function of $D$.

$$
H=\frac{S \cot A}{2}\left[1-\left(1-\frac{4 D}{S}\left(1+\frac{D}{S}\right) \tan ^{2} A\right)\right]^{\frac{1}{2}}
$$

Note that this result involves the parameters $H, S$ and $D$ only in the ratios $H / S$ and $D / S$, so that it is really a three-parameter relation giving $H / S$ in terms of $D / S$ and $A$.

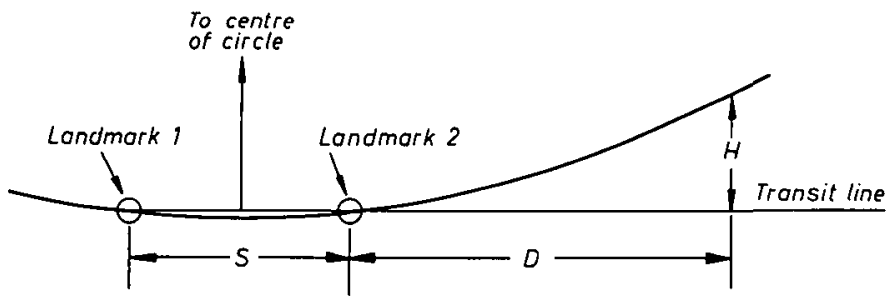

Fig. I. Parameters $H, S$ and $D$ that define a small angle circle as offsets from the transit line

For the proof of ( 1 ) we first derive an auxiliary result involving the right-angled triangle in Fig. 2 for which one side is the length $R \cos A$ and $R+L$ is the hypotenuse. One point about the figure should be noted here to avoid confusion. The angle $A$ is defined as the angle subtended by the two landmarks from a point on the circumference of the circle. That angle is not shown in Fig. 2. But it can be proved that it is the same size as the 


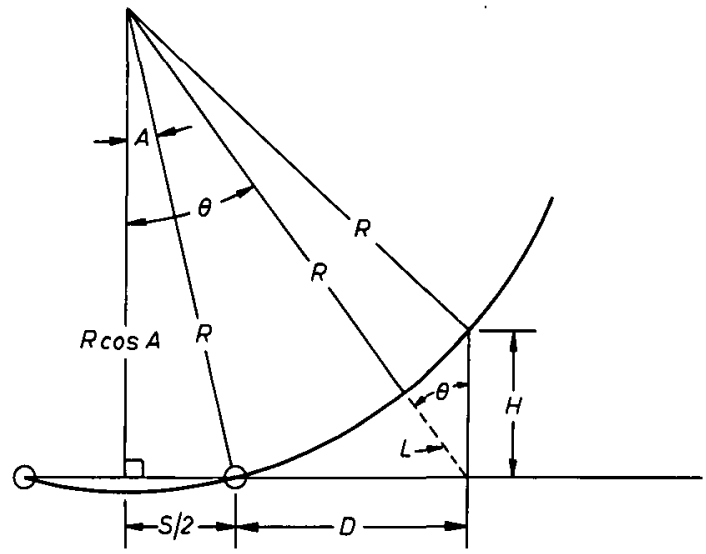

Fig. 2. Construction for the proof of equation (I)

angle subtended at the centre of the circle by the mid point between the two landmarks and one of the landmarks themselves. This is the angle $A$ that is shown in the figure.

For proving (1) we first refer to Fig. 2, where we see that

$$
R^{2} \cos ^{2} A+\left(\frac{S}{2}+D\right)^{2}=(R+L)^{2}
$$

or

$$
R^{2}-R^{2} \sin ^{2} A+\frac{S^{2}}{4}+S D+D^{2}=R^{2}+2 R L+L^{2}
$$

Since $R \cdot \sin A=S / 2$ this equation simplifies to

$$
2 R L+L^{2}=S D+D^{2}
$$

Now refer to the acute triangle in this figure with sides of length $R+L, H, R$. From the law of cosines,

$$
R^{2}=(R+L)^{2}+H^{2}-2(R+L) H \cos \theta
$$

Use $\cos \theta=R \cos A /(R+L)$, which is obvious from Fig. 2, and this equation becomes

$$
0=2 R L+L^{2}+H^{2}-2 H R \cos A
$$

Combining this result with equation (2) we have

or

$$
\begin{gathered}
H^{2}-2 H R \cos A+S D+D^{2}=0 \\
H=R \cos A \pm\left(R^{2} \cos ^{2} A-\left(S D+D^{2}\right)\right)^{\frac{1}{2}}
\end{gathered}
$$

We take the minus sign so that $H \rightarrow 0$ when $D \rightarrow 0$ and find

$$
H=R \cos A\left(1-\left(1-\frac{S D+D^{2}}{R^{2} \cos ^{2} A}\right)^{\frac{1}{2}}\right)
$$

Now we use $R=S / 2 \sin A$ to derive equation (1).

A remark on the validity of equation ( 1 ) is here added. For the value of $D$ shown in Fig. 2 the circle arc is more or less horizontal, but for larger and larger $D$ the arc continues to turn up and at one point it becomes vertical. This happens when $D+S / 2=R$, and for larger values of $D$ than that defined by this relation equation ( 1 ) ceases to be meaningful. 
Although equation ( 1 ) is exact it does require computation and is not particularly transparent. An approximation to it which is more intuitive is often adequate. To derive it write (1) as

where

$$
H / S=(\cot A / 2)\left(1-[1-\lambda]^{\frac{1}{2}}\right)
$$

$$
\lambda=4(D / S)(1+D / S) \tan ^{2} A \approx(D / S)(1+D / S) 1 \cdot 218 \times 10^{-3}\left(A^{\circ}\right)^{2}
$$

The approximate form for $\lambda$ on the extreme right-hand side of equation (3) is derived by using the fact that $\tan A \approx A$ for small $A$ and converting $A$ (in radians) to $A^{\circ}$ (in degrees) by $A=A^{\circ} / 57 \cdot 3=0.0175 A^{\circ}$. This form is accurate to better than $\frac{1}{2}$ per cent for $A$ less than $10^{\circ}$. With this form, and the fact that for $\lambda$ small, which it usually is, $(1-\lambda)^{\frac{1}{2}} \approx 1-\lambda / 2$, we find

$$
H / S \approx 0.01745(D / S)(1+D / S) A^{\circ}
$$

Table I compares the accuracy of this approximation with the exact equation ( 1 ).

Table 1. Exact values of H/S From equation (i) versus approximate ones from EQUATION (4)

\begin{tabular}{rllllll}
\hline & \multicolumn{2}{c}{$D / S=1.0$} & \multicolumn{2}{c}{$D / S=2.0$} & \multicolumn{1}{c}{$D / S=3.0$} \\
\cline { 2 - 7 }$A^{0}$ & Exact & Approx. & Exact & Approx. & Exact & Approx. \\
\hline 1 & 0.0349 & 0.0349 & 0.105 & 0.105 & 0.210 & 0.209 \\
2 & 0.0699 & 0.0699 & 0.211 & 0.209 & 0.425 & 0.418 \\
3 & 0.105 & 0.105 & 0.320 & 0.314 & 0.651 & 0.627 \\
4 & 0.141 & 0.140 & 0.433 & 0.418 & 0.895 & 0.836 \\
5 & 0.178 & 0.175 & 0.552 & 0.525 & 1.169 & 1.045 \\
6 & 0.215 & 0.209 & 0.679 & 0.630 & 1.497 & 1.254 \\
7 & 0.253 & 0.244 & 0.819 & 0.735 & 1.932 & 1.463 \\
8 & 0.293 & 0.279 & 0.978 & 0.840 & 2.747 & 1.680 \\
9 & 0.334 & 0.314 & 1.165 & 0.945 & & \\
10 & 0.378 & 0.349 & 1.408 & 1.047 & & \\
\hline
\end{tabular}

3. ACCURACY OF TRANSIT LINES OF POSITION. The formulae above have a variety of applications, one of which, the question of the accuracy of transit lines, is now discussed.

Two objects are said to be in transit when we judge the angle between them to be zero. But our judgment is not perfect, and when the eye says the angle is zero a more accurate instrument might say it is merely small. What error of position does this imply? There are really two questions here. How accurate is the estimate of zero angle? What is the effect on position determination of the error in the estimate?

The first question has no general answer. The accuracy of determining that two objects are exactly in transit depends on the nature of the objects, the degree of clarity of the viewing conditions and the observer's visual acuity, among other factors. The accuracy is usually a small fraction of a degree, but whether that fraction is a tenth or a fiftieth cannot be answered in general. This question is really the classical problem (with additional complications) of the resolving power of the eye in ideal circumstances, i.e. when the resolving power is determined by the eye itself and is not limited by poor lighting, hazy atmospheric conditions, etc. In this ideal case the resolving power is usually taken to be one minute of arc (one-sixtieth of a degree), the angular separation at which two separate points begin to appear distinct. Another kind of resolving power is vernier 
acuity, the ability to align two straight-line segments, a line and a cross hair, etc. Vernier acuity is often several times better than visual acuity, so that some observers can repeat a vernier setting to a tenth of a minute.

These limits are useful to know, since one cannot hope to improve on them in transit determinations. In most practical cases of course one will do worse, since the objects in question - tanks, cupolas, lighthouses, etc. - are not so clearcut visually. Unlike lines or points they may have a substantial angular width, so that one has to estimate the position of the centre of each object as well as estimating when these centres are lined up; or they may not be in the same horizontal plane (standpipe on a hill and a buoy in the water); or the observation deck or platform may be moving, etc.

TABle 2. POSITION ERRORS IN FEET THAT ARISE IF TWO LANDMARKS ARE ERRONEOUSLY CONSIDERED TO BE IN TRANSIT WHEN IN FACT THERE IS AN ANGLE OF $O \cdot 1^{\circ}$ BETWEEN THEM

\begin{tabular}{|c|c|c|c|c|}
\hline & & \multicolumn{3}{|c|}{$\begin{array}{c}\text { Distance } D \text { from nearer } \\
\text { landmark (n.m.) }\end{array}$} \\
\hline & & 1 & 2 & 3 \\
\hline \multirow{3}{*}{$\begin{array}{l}\text { Spacing } S \\
\text { of landmarks } \\
\text { (n.m.) }\end{array}$} & I & $2 \mathrm{I}$ & 37 & I 27 \\
\hline & 2 & 64 & 42 & 35 \\
\hline & 3 & 127 & 79 & 63 \\
\hline
\end{tabular}

As a crude guess in average conditions I shall initially assume an error of $0.1^{\circ}$, which is six times the ideal resolving power for two points. This figure results from some simple experiments that I have tried, and it is in no way definitive. I simply use it as a substitute for $x$ in the question: if the error in a transit determination is $x$ degrees, what is the resulting error in a position determination based on that transit? This question can be answered with the help of equation (4). Suppose for example that the two landmarks are a mile apart $(S=I$ mile) and that the observer is one mile from the nearer one $\left(D=\mathrm{I}\right.$ mile). The observer thinks he is on a perfect transit line, $A=0.0^{\circ}$, when in fact he is on a small angle COP for which $A=0.1^{\circ}$. The error in position due to the error in angle, i.e. the distance $H$ from the transit line, is given by equation (4), with $D / S=1 \cdot 0, S=1 \cdot 0$ and $A=0.1$, and turns out to be just $21 \mathrm{ft}$. A variety of similar examples is provided in Table 2 . If the angular error were $0.2^{\circ}$ instead of $0.1^{\circ}$ in the examples above the distance error would of course be doubled.

As examples with increased accuracy one might imagine a particularly favourable natural transit, perhaps a slim stack and a flagpole on a very clear day. Or with some thought it might be possible to design artificial leading marks with narrow vertical lines that exploit the vernier acuity mentioned above. In such cases it might then be feasible to determine transit to within a minute of arc. If this were so the errors in the above table would be reduced to one-sixth of the values shown, so that in some cases they would be only of the order of a few feet.

\section{REFERENCES}

1 See for example any book that discusses coastal navigation such as Norman Dahl's The Yacht Navigator's Handbook (Ward Lock, London, 1983 ).

2 Eyges, L. (1987). Safety in Circles. Navigation News 2, 15. 